\title{
Precise large deviation of the surplus process in a perturbed model
}

\author{
Yinghua Dong \\ College of Mathematics and Statistics, Nanjing University of Information \\ Science and Technology, Nanjing, 210044, China \\ dongyinghua1@163.com
}

\begin{abstract}
.
In this paper, we consider a perturbed model in which $\left\{X_{i}, i=1,2, \cdots\right\}$ are extended negatively dependent random variables with consistently varying tails, $\left\{Y_{k}, k=1,2, \cdots\right\}$ are idependent, identically distributed random variables. We give precise large deviation of the surplus process .
\end{abstract}

Keywords: Precise large deviation, the surplus process, extended negative dependence, quasi-renewal process.

\section{Introduction}

We consider a model in which $\left\{X_{i}, i=1,2, \cdots\right\}$ form a sequence of nonnegative extended negative dependent (END) random variables with common distribution $F ; N(t)$ denotes the appearance number of $\left\{X_{i}, i=1,2, \cdots\right\}$ in $[0, t]$, and $\{N(t), t \geq 0\}$ is a general counting process. We assume $E(N(t))=\Lambda(t)<\infty$ for all $t \geq 0$, and as $t \rightarrow \infty$, $\Lambda(t) \rightarrow \infty$. The aggregate amount up to $t$ can be given by

$$
S(t)=\sum_{i=1}^{N(t)} X_{i}, \quad t \geq 0 .
$$

$\left\{Y_{k}, k=1,2, \cdots\right\}$ constitute another sequence of independent, identically distributed (i.i.d) nonnegative random variables. Suppose that their inter-arrival time $\left\{\theta_{i}, i=1,2, \cdots\right\}$ forms a sequence of identically distributed LND random 
variables. Let $T_{k}=\sum_{i=1}^{k} \theta_{i}$ denote the arrival time of $Y_{k}$. Then we get a quasi-renewal process $M(t)=\sup \left\{n \geq 1: T_{n} \leq t\right\} \quad, \quad t \geq 0$. Let $E\left(\theta_{1}\right)=1 / \lambda_{1}$. Then $M(t) / \lambda_{1} t \longrightarrow 1$, a.e.

Let $u>0$ denote the initial reserve. $\{\sigma W(t), t \geq 0\}$ denotes a perturb process, where $\sigma$ is referred to as a diffusion coefficient, and $\{W(t), t \geq 0\}$ is a standard Wiener process. Let $d$ and $-d$ denote the upper and low bounds of $\{W(t), t \geq 0\}$, respectively. The reserve process is presented by

$$
R(t)=u+\sum_{k=1}^{M(t)} Y_{k}-\sum_{i=1}^{N(t)} X_{i}+\sigma W(t) I_{[-d, d]}(W(t)), \quad t \geq 0 .
$$

The surplus process can be denoted by

$$
Z(t)=\sum_{i=1}^{N(t)} X_{i}-\sum_{k=1}^{M(t)} Y_{k}-\sigma W(t) I_{[-d, d]}(W(t)), \quad t \geq 0 .
$$

we assume $\left\{X_{i}, i=1,2, \cdots\right\},\left\{Y_{k}, k=1,2, \cdots\right\},\{N(t), t \geq 0\} \quad$ and $\{M(t), t \geq 0\}$ are mutually independent. [1] showed precise large deviation of non-random sum, while [2] presented precise large deviation of $\{S(t), t \geq 0\}$. In the present paper, we obtain precise large deviation of the surplus process $\{Z(t), t \geq 0\}$ in the above model.

\section{Preliminaries}

The definition of END structure was introduced by [1].

Definition 1. We call random variables $\left\{X_{i}, i=1,2, \cdots\right\}$ END if there is $M>0$ such that both $P\left\{X_{1} \leq x_{1}, X_{2} \leq x_{2}, \cdots, X_{n} \leq x_{n}\right\} \leq M P\left\{X_{1} \leq x_{1}\right\} P\left\{X_{2} \leq x_{2}\right\} \cdots P\left\{X_{n} \leq x_{n}\right\}$ 
and

$P\left\{X_{1}>x_{1}, X_{2}>x_{2}, \cdots, X_{n}>x_{n}\right\} \leq M P\left\{X_{1}>x_{1}\right\} P\left\{X_{2}>x_{2}\right\} \cdots P\left\{X_{n}>x_{n}\right\}$

hold for each $n=1,2, \cdots$, and all $x_{1}, \cdots x_{n}$. For $M=1$, if (2) holds, we call $\left\{X_{i}, i=1,2, \cdots\right\}$ LND.

In the following, we introduce some related heavy-tailed distribution class, which can be found in [3] and [4]. For convenience, denote $\bar{F}(x)=1-F(x)=P(X>x)$.

A distribution $F$ on $[0, \infty)$ is said to belong to the long-tailed class and write $F \in L$, if

$$
\bar{F}(x-y) \sim \bar{F}(x), \quad \text { for any } y \in(-\infty, \infty) .
$$

In addition, we say that $F$ is said to belong to the dominated variation class and written as $F \in D$, if

$$
\bar{F}(x y)=O(1) \bar{F}(x) \text {, for all } 0<y<1 .
$$

Denote the upper Matuszewska index of $F$ by $J_{F}^{+}$. If $F \in D$, then $0<J_{F}^{+} \leq \infty$.

The consistent variation class $C$ is smaller than the class $D$. We call $F \in C$, if

$$
\lim _{y \downarrow 1} \liminf _{x \downarrow \infty} \frac{\bar{F}(x y)}{\bar{F}(x)}=1 .
$$

It is well known that $C$ belongs to $D \cap L$.

In this paper, $N(t)$ satisfies the following assumption.

Assumption 1: For some $p>J_{F}^{+}$,

$$
E N(t)^{p} I(N(t)>(1+\delta) \Lambda(t))=O(1) \Lambda(t)
$$

holds for all $\delta>0$. 
According to Theorem 3.1 of [2], we can get the following lemma.

Lemma 1. Let $\left\{X_{i}, i=1,2, \cdots\right\}$ be END. In addition to Assumption 1, suppose that $F \in C, E\left(X_{1}\right)=\mu>0$. Then, for any fixed $\gamma>0$, it holds uniformly for all $x \geq \gamma \Lambda(t)$ that

$$
P(S(t)-\mu \Lambda(t)>x) \sim \Lambda(t) \bar{F}(x), \quad t \rightarrow \infty .
$$

By the definition of the consistent variation class $C$, we easily get the following lemma.

Lemma 2. If $F \in C$, then

$$
\lim _{x \rightarrow \infty} \frac{\bar{F}(x+o(1) x)}{\bar{F}(x)}=1 .
$$

The following lemma is due to [5]

Lemma 3. For a distribution $F$ on $[0, \infty)$, if $F \in D$, then for any $p>J_{F}^{+}$, there exists some positive number $C_{1}$ and $x_{0}$ such that

$$
\frac{\bar{F}(x y)}{\bar{F}(x)} \leq C_{1} y^{-p}, \quad \text { for all } x \geq x y \geq x_{0} .
$$

The following is from [2].

Lemma 4. For a qusi-renewal process $\{M(t), t \geq 0\}$, the generic inter-renewal distance $\theta$ has distribution $G$ and expectation $1 / \lambda_{1}<\infty$. If $G(\infty)=1$, then

$$
\lim _{t \rightarrow \infty} \frac{\lambda_{1}(t)}{t}=\lambda_{1}, \quad \text { a.e. }
$$

holds.

\section{Main result}


Theorem. Let $\left\{X_{i}, i=1,2, \cdots\right\}$ be END. In addition to Assumption 1, suppose that $F \in C, E\left(X_{1}\right)=\mu>0$. Then for any fixed $\gamma>0$, it holds uniformly for all $x \geq \gamma \Lambda(t) \geq \lambda_{1} t$ satisfying $\gamma \geq E\left(Y_{1}\right)$ that

$$
P(Z(t)-\mu \Lambda(t)>x) \sim \Lambda(t) \bar{F}(x), \quad t \rightarrow \infty .
$$

Proof. For convenience, write $A(t)=\sum_{i=1}^{M(t)} Y_{i}+\sigma W(t) I_{[-d, d]}(\sigma W(t))$.

$$
\text { Since } E\left(\sum_{k=1}^{M(t)} Y_{k}\right)=E\left(Y_{1}\right) \lambda_{1}(t) \text { and } E\left(W(t) I_{[-d, d]}(\sigma W(t))\right)=0 \text {, }
$$

We get $E(A(t))=E\left(Y_{1}\right) \lambda_{1}(t)$. According to Chen et al. (2011), we have

$$
\frac{1}{E\left(Y_{1}\right) \lambda_{1} t} \sum_{k=1}^{M(t)} Y_{k}=\frac{1}{M(t)} \sum_{k=1}^{M(t)} Y_{k} \cdot \frac{M(t)}{\lambda_{1} t} \longrightarrow 1, \quad \text { a.e. }
$$

In addition, it is clear that

$$
\lim _{t \rightarrow \infty} \frac{W(t) I_{[-d, d]}(W(t))}{t}=0,
$$

It follows from the two equalities above that

$$
\frac{A(t)-E\left(Y_{1}\right) \lambda_{1}(t)}{E\left(Y_{1}\right) \lambda_{1} t} \longrightarrow 0,
$$

Hence, there is a positive function $\varepsilon(t)$ such that as $t \rightarrow \infty, \varepsilon(t) \rightarrow 0$ and

$$
P\left(\left|A(t)-E\left(Y_{1}\right) \lambda_{1}(t)\right|>\varepsilon(t) E\left(Y_{1}\right) \lambda_{1} t\right)=o(1) .
$$

Next we discuss the large deviation of the surplus process.

$$
\begin{aligned}
& P(Z(t)-E Z(t)>x) \\
= & P\left(S(t)-A(t)-E S(t)+E\left(Y_{1}\right) \lambda_{1}(t)>x\right) \\
\quad= & \int_{\left|y-E\left(Y_{1}\right) \lambda_{1}(t)\right| \varepsilon \varepsilon(t) E\left(Y_{1}\right) \lambda_{1} t} P\left(S(t)-E S(t)>x-E\left(Y_{1}\right) \cdot \lambda_{1}(t)+y\right) P(A(t) \in d y)
\end{aligned}
$$




$$
\begin{aligned}
& \quad+\int_{y-E\left(Y_{1}\right) \lambda_{1}(t)<-\varepsilon(t) E\left(Y_{1}\right) \lambda_{1} t} P\left(S(t)-E S(t)>x-E\left(Y_{1}\right) \cdot \lambda_{1}(t)+y\right) P(A(t) \in d y) \\
& +\int_{y-E\left(Y_{1}\right) \lambda_{1}(t)>\varepsilon(t) E\left(Y_{1}\right) \lambda_{1} t} P\left(S(t)-E S(t)>x-E\left(Y_{1}\right) \cdot \lambda_{1}(t)+y\right) P(A(t) \in d y) \\
& =I_{1}(t)+I_{2}(t)+I_{3}(t) .
\end{aligned}
$$

First of all, we deal with $I_{1}(t)$. For $x \geq \gamma \Lambda(t) \geq \lambda_{1} t$, we have $\frac{t}{x} \leq \frac{1}{\lambda_{1}}$

When $\quad\left|y-E\left(Y_{1}\right) \cdot \lambda_{1}(t)\right| \leq \varepsilon(t) E\left(Y_{1}\right) \lambda_{1} t \quad$, $x-E\left(Y_{1}\right) \lambda_{1}(t)+y=x+o(1) t=x+o(1) x$.

By Lemma 1 and Lemma 2, it holds uniformly all $x \geq \gamma \Lambda(t)$,

$$
\begin{aligned}
& I_{1}(t) \\
& =\int_{\left|y-E\left(Y_{1}\right) \lambda_{1}(t)\right| \leq \varepsilon(t) E\left(Y_{1}\right) \lambda_{1} t} P\left(S(t)-E S(t)>x-E\left(Y_{1}\right) \cdot \lambda_{1}(t)+y\right) P(A(t) \in d y)
\end{aligned}
$$

$$
\begin{aligned}
& \sim \int_{\left|y-E\left(Y_{1}\right) \lambda_{1}(t)\right| \leq \varepsilon(t) E\left(Y_{1}\right) \lambda_{1} t} \Lambda(t) \bar{F}\left(x-E\left(Y_{1}\right) \cdot \lambda_{1}(t)+y\right) P(A(t) \in d y) \\
& =\Lambda(t) \bar{F}(x) \int_{\left|y-E\left(Y_{1}\right) \lambda_{1}(t)\right| \leq \varepsilon(t) E\left(Y_{1}\right) \lambda_{1} t} \frac{\bar{F}(x+o(1) x)}{\bar{F}(x)} P(A(t) \in d y) \\
& \sim \Lambda(t) \bar{F}(x) .
\end{aligned}
$$

Next we discuss $I_{2}(t)$. By Lemma 3, there is some positive number $D_{2}$ such that it holds uniformly for all $x \geq \gamma \Lambda(t)$ satisfying $\gamma>E\left(Y_{1}\right)$, 


$$
\begin{aligned}
& I_{2}(t)=\int_{y-E\left(Y_{1}\right) \lambda_{1}(t)<-\varepsilon(t) E\left(Y_{1}\right) \lambda_{1} t} P\left(S(t)-E S(t)>x-E\left(Y_{1}\right) \lambda_{1}(t)+y\right) P(A(t) \in d y) \\
& \leq \int_{y-E\left(Y_{1}\right) \lambda_{1}(t)<-\varepsilon(t) E\left(Y_{1}\right) \lambda_{1} t} P\left(S(t)-E S(t)>x-E\left(Y_{1}\right) \cdot \lambda_{1}(t)\right) P(A(t) \in d y) \\
& \begin{array}{r}
\sim(t) \bar{F}(x) \int_{y-E\left(Y_{1}\right) \lambda_{1}(t)<-\varepsilon(t) E\left(Y_{1}\right) \lambda_{1} t} \frac{\bar{F}\left(x-E Y_{1} \lambda_{1}(t)\right)}{\bar{F}(x)} P(A(t) \in d y) \\
\leq D_{2} \Lambda(t) \bar{F}(x) P\left(A(t)-E\left(Y_{1}\right) \cdot \lambda_{1}(t)<-\varepsilon(t) E\left(Y_{1}\right) \lambda_{1} t\right) \\
=o(1) \Lambda(t) \bar{F}(x) .
\end{array}
\end{aligned}
$$

Now we verify the fourth step. As $t$ is large enough and $x \geq \gamma \Lambda(t)$,

$$
x-E\left(Y_{1}\right) \Lambda(t)=x\left(1-\frac{E\left(Y_{1}\right) \cdot \Lambda(t)}{x}\right) \geq x\left(1-\frac{E\left(Y_{1}\right)}{\gamma}\right)
$$

Since $C \subset D$,

$$
\limsup _{x \rightarrow \infty} \frac{\bar{F}\left(x\left(1-\frac{E\left(Y_{1}\right)}{\gamma}\right)\right)}{\bar{F}(x)}<\infty
$$

Hence, as $t$ is enough large, there is some positive number $D_{2}$ such that

$$
\frac{\bar{F}\left(x-E\left(Y_{1}\right) \Lambda(t)\right)}{\bar{F}(x)} \leq D_{2} .
$$

Finally, we deal with $I_{3}(t)$. For any fixed $\gamma>0$, it holds uniformly for all $x \geq \gamma \Lambda(t)$ that 


$$
\begin{aligned}
I_{3}(t)=\int_{y-E\left(Y_{1}\right) \lambda_{1}(t)>\varepsilon(t) E\left(Y_{1}\right) \lambda_{1} t} P\left(S(t)-E S(t)>x-E\left(Y_{1}\right) \cdot \lambda_{1}(t)+y\right) P(A(t) \in d y) \\
\quad \leq \int_{y-E\left(Y_{1}\right) \lambda_{1}(t)>\varepsilon(t) E\left(Y_{1}\right) \lambda_{1} t} P(S(t)-E S(t)>x) P(A(t) \in d y) \\
\quad \sim \Lambda(t) \bar{F}(x) P\left(A(t)-E\left(Y_{1}\right) \lambda_{1}(t)>\varepsilon(t) E\left(Y_{1}\right) \lambda_{1} t\right) \\
\quad=o(1) \Lambda(t) \bar{F}(x) .
\end{aligned}
$$

According to (4)-(7), we obtain (3). This ends the proof of the theorem.

\section{Reference:}

[1] Y. Liu, Precise large deviations for dependent random variables with heavy tails. Stat.

Probab.Lett. , 47, (2004) 311-319.

[2] Y. Chen, K. C. Yuen and K. W. Ng, Precise large deviations of random sums in the presence of

negatively dependence and consistent variation. Methodol. Comput. Appl. Probab. 13 (2011) 821-833.

[3] N. H. Bingham, C. M. Glodie, and J. L. Teugels, Regular Variation, Cambridge University

Press. (1987).

[4] D. B. H. Cline and Samorodnitsky G, Subexponentiality of the product of independent random variables, Stoch. Proc. Appl. 49, (1994) 75-98.

[5] Q. Tang and G. Tsitsiashvili, Precise estimates for the ruin probability in finite horizon in a discrete-time model with heavy-tailed insurance and financial risks. Stoch. Proc. Appl. 108, (2003) 299-325. 